\title{
Détermination d'un Profil Agroforestier Local à Base de Cacaoyers dans les Environs de Kokumbo au Centre de la Côte d'Ivoire
}

\author{
Kouamé Kra Modeste Adingra,
}

UFR Sciences de l'Homme et de la Société (SHS),

Université Félix Houphouët-Boigny Abidjan, Côte d'Ivoire

Kouadio Venance-Pâques Gniayou,

Laboratoire de Botanique, UFR Biosciences, Université Félix Houphouët-Boigny Abidjan, Côte d'Ivoire

\section{Fulbert Tra,}

UFR Sciences de l'Homme et de la Société (SHS), Université Félix Houphouët-Boigny Abidjan, Côte d'Ivoire

\section{Doi:10.19044/esj.2020.v16n6p541～URL:http://dx.doi.org/10.19044/esj.2020.v16n6p541}

\section{Résumé}

Le présent article porte sur l'émergence d'un profil agroforestier local à base de cacaoyers dans les environs de Kokumbo. Les paysans, dans cette partie de la Côte d'Ivoire optent depuis des années pour l'exploitation agroforestière à base de cacaoyers, une pratique agricole durable encouragée par l'Etat et les acteurs environnementaux. La méthodologie adoptée est basée sur une approche qualitative combinant la revue documentaire, les interviews semi-structurées et l'observation participante. Au total 32 agroforestiers habitant les villages de Niamkey-Konankro et de Langossou ont été interrogés à l'aide des guides d'entretien préétablis. Les résultats obtenus montrent que la pratique agroforestière à base de cacaoyers dans cette partie du pays repose sur des fondements socioculturels et politiques solides qui engraissent sa continuation comme option de cacaoculture privilégiés par les locaux.

Mots-clés : Agroforesterie, habitus cacaoculturaux, développement durable, Kokumbo, Côte d'Ivoire 


\title{
Determination of a Local Cocoa-based Agroforestry Profile in Kokumbo Vicinity in the Centre of Côte d'Ivoire
}

\author{
Kouamé Kra Modeste Adingra, \\ UFR Sciences de l'Homme et de la Société (SHS), \\ Université Félix Houphouët-Boigny Abidjan, Côte d'Ivoire \\ Kouadio Venance-Paques Gniayou, \\ Laboratoire de Botanique, UFR Biosciences, \\ Université Félix Houphouët-Boigny Abidjan, Côte d'Ivoire

\section{Fulbert Tra,} \\ UFR Sciences de l'Homme et de la Société (SHS), \\ Université Félix Houphouët-Boigny Abidjan, Côte d'Ivoire
}

\begin{abstract}
This paper focuses on the development of a local cocoa-based agroforestry profile in Kokumbo vicinity. The farmers in this part of the Côte d'Ivoire have been opting for a cocoa-based agroforestry system for years. This system is a sustainable agricultural practice encouraged by the government and environmental actors. This study is based on a qualitative approach that combines document analysis, semi-structured interviews and participant observation. A total amount of 32 agroforestry smallholders living in the villages of Niamkey-Konankro and Langossou were interviewed using pre-established interview guides. The results have showed that the cocoabased agroforestry practice in this part of the country lies on solid sociocultural and political foundations which encourage its adoption as a cocoagrowing option favored by the local populations.
\end{abstract}

Keywords: Agroforestry, Cocoa-Cultural Habitus, Sustainable Development, Kokumbo, Côte d'Ivoire

\section{Introduction}

L'économie ivoirienne repose en grande partie sur sa production cacaoyère (Adou Yao et al., 2016). En effet, une décennie après son accession à l'indépendance, les partenaires internationaux du monde économique et financier félicitaient la Côte d'Ivoire d'avoir réalisé un «miracle économique » (ICCO, 2008). Cet essor économique est dû à la culture du 
cacao qui a produit 1,8 million de tonnes en 2015. Actuellement, la culture du cacao contribue pour $10 \%$ à la formation du Produit Intérieur Brut (PIB) et fournit $40 \%$ des recettes d'exportation (cacao.gouv.ci). Au niveau politicoéconomique, la promotion des variétés améliorées de cacao telles que Mercedes conjuguée à la recherche de la rentabilité économique stricto sensu des exploitations cacaoyères a eu pour conséquence l'expansion de la cacaoculture.

Le développement de cette monoculture peu soucieuse de l'environnement s'est traduit par une dégradation du couvert forestier ivoirien (Adou Yao et al., 2016). Pendant longtemps, la Côte d'Ivoire a profité des largesses de la nature en exploitant sans discontinuer la forêt. Le rang de premier producteur mondial de fèves de cacao s'est construit au détriment de cette exploitation forestière (Kouadio et al., 2007). Pour faire face à la régression progressive du couvert forestier ivoirien, une des alternatives recommandées c'est l'agroforesterie. C'est un ensemble de systèmes d'utilisation des terres où des plantes ligneuses pérennes (arbres, arbustes) croissent en association avec des plantes herbacées (cultures, pâturages) ou le bétail, en arrangement spatial, en rotation, ou les deux.

L'agroforesterie propose des éléments de réponses à cette mauvaise situation puisqu'elle vise à la fois la production agricole et la production forestière (Seif El Din, 1981). Depuis des décennies, les systèmes agroforestiers cacaoyers fondés sur des connaissances locales, contribuent à la satisfaction des besoins des paysans et des exigences écologiques en Afrique. Dans l'Etat d'Ondo au Nigeria, l'évaluation de la valeur de conservation des agroforêts est abordée par Oke et al. (2007). Les auteurs postulent que les agroforêts à cacaoyers traditionnelles ont contribué à la conservation des essences forestières. En effet, les systèmes agroforestiers ont, selon ces auteurs, non seulement la capacité de conserver la plupart des espèces d'arbres forestiers, mais aussi le pouvoir de produire du bois et des fruits comestibles.

Aussi, Luke et al. (2011) évaluent-ils l'impact de la cacaoculture sur la diversité des arbres. Ils notent que les jachères et les fermes de cacaoyers au Ghana constituent un important réservoir de biodiversité. Lequel est capable, de fournir une orientation pour la conservation de certaines espèces d'arbres menacées et vulnérables. Les auteurs attribuent la régénération et le rétablissement de ces espèces dans les jachères aux connaissances locales de la gestion de l'ombre.

L'agroforesterie à base de cacaoyers est une pratique ancienne et ancestrale en Côte d'Ivoire. Elle est utilisée comme alternative aux choix agricoles destructeurs de la forêt des paysans. La Côte d'Ivoire, premier producteur mondial de cacao devrait intégrer le concept de développement durable dans les activités de la cacaoculture afin de pérenniser sa productivité et conserver son rang dans la commercialisation des fèves de cacao. Les 
villages de Niamkey-Konankro et de Langossou appartenant au département de Kokumbo sont situés au Centre de la Côte d'Ivoire et sont le site de développement d'un système agroforestier à base de cacaoyers traditionnel. Ce système agroforestier intéresse les scientifiques du fait de sa durabilité et sa stabilité (Photo 1).

L'objectif de ce travail est d'exposer les fondements socioanthropologiques de la durabilité agroforestière à base de cacaoyers à travers la détermination du profil agroforestier à base de cacaoyers local, base de données pour les acteurs d'environnement pour repenser la cacaoculture en Côte d'Ivoire.

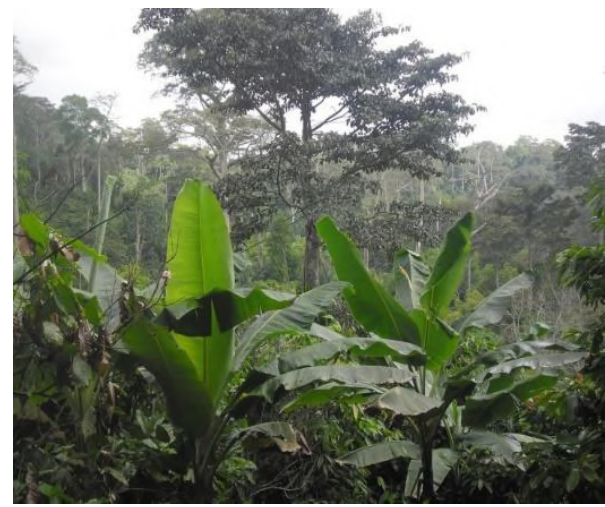

Photo 1. Agroforêt à base de cacaoyers installée en zone forestière à Niamkey-Konankro, Centre de la Côte d'Ivoire

\section{Matériels et méthodes}

\subsection{Présentation de la zone d'étude}

L'étude a été conduite au centre de la Côte d'Ivoire dans les villages de Niamkey-Konankro et de Langossou de la sous-préfecture de Kokumbo (Figure 1). Les populations de la zone d'étude sont majoritairement composées d'autochtones baoulé. On y rencontre très peu de populations étrangères.

\subsection{Collecte des données}

L'approche méthodologique utilisée dans cette étude est de type qualitatif. Elle a consisté en l'étude des savoirs, savoir-faire, pratiques et connaissances agricoles des populations locales. Pour cela, nous nous sommes appuyés sur la théorie de l'habitus de Bourdieu qui s'inscrit dans le paradigme constructiviste (Bourdieu, 1980). Selon cette théorie, les savoirs agricoles et les techniques cacaoculturales sont acquis au cours du processus de socialisation des paysans. Aussi, cette réalité devient une forme de programmation agricole. A cet effet, le paysan sait la méthode ou la technique pour établir son champ de cacao qui est agroforêt à base de cacaoyers. 
En pratique, la collecte des données a nécessité l'utilisation de la revue documentaire, des interviews semi-structurés et de l'observation participante. Les interviews semi-structurées ont été réalisées auprès des agroforestiers des villages de Niamkey-Konankro et de Langossou avec l'aide d'un guide d'entretien. Au total, 32 individus (27 cacaoculteurs, 3 ANADER et 2 BIOPARTENAIRE) ont été interrogés au cours de cette étude. Le choix des personnes était fondé sur deux (2) critères : habiter l'un des deux villages et pratiquer la cacaoculture puis le statut dans le groupe du répondant. Cependant, la taille de notre échantillon a été déterminée en appliquant la technique de la saturation car à partir de 32 individus, les données recueillies ne fournissaient plus d'éléments nouveaux à l'étude.

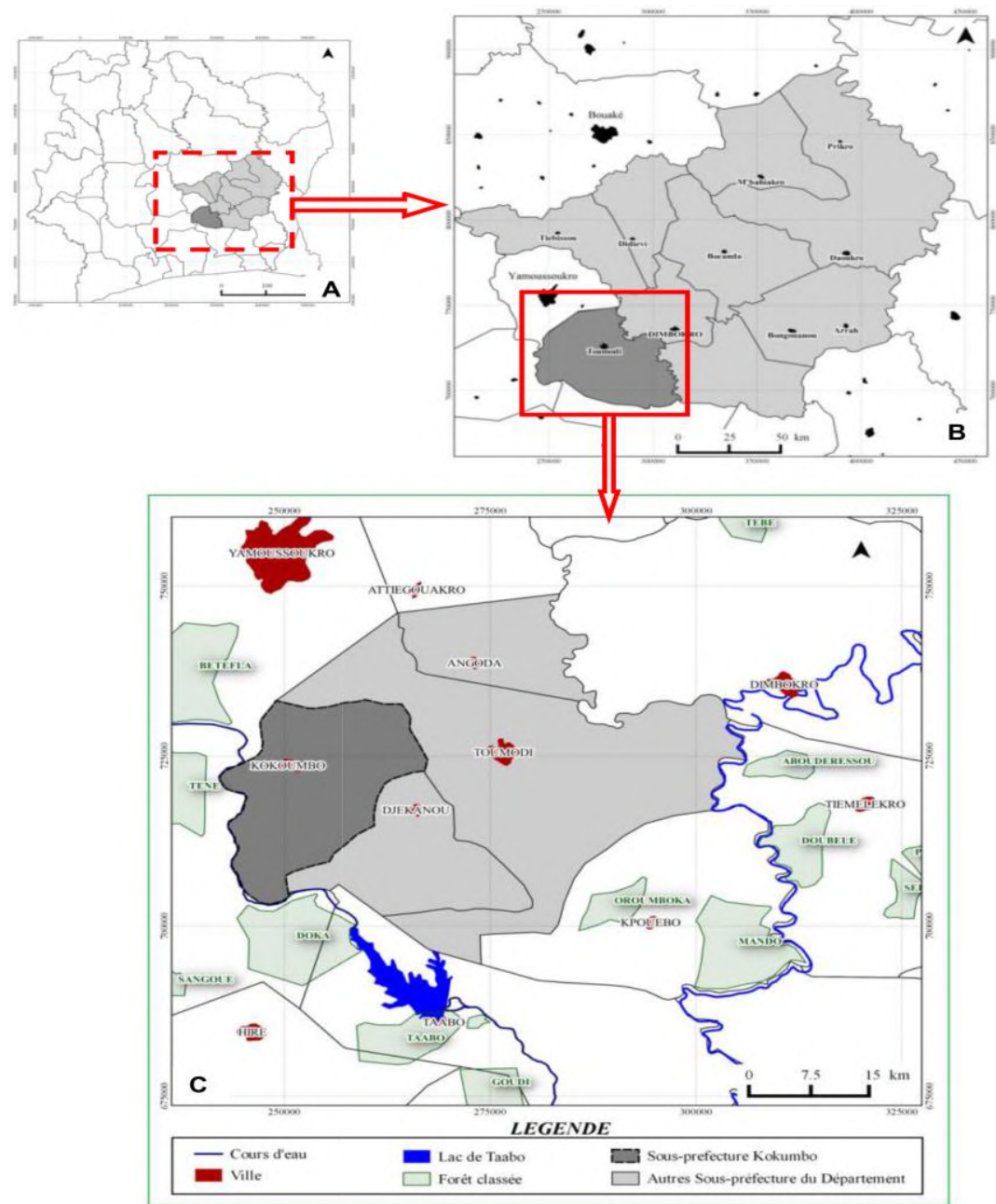

Figure 1. Localisation de la sous-préfecture de Kokumbo (Kpangui, 2015)

A : District des lacs,

B : Département de Toumodi,

C : Sous-préfecture de Kokumbo 
Enfin, nous avons fait une immersion dans l'univers des populations locales pendant deux (2) mois. Cela nous a permis de les observer et de les écouter suivant leurs représentations sociales en lien avec la culture du cacaoyer.

\subsection{Analyse des données}

Après la réalisation de tous les entretiens, les guides d'entretien ont été rassemblés et codifiés puis dépouillés manuellement. Cette opération avait pour but d'isoler chaque entretien afin d'en faciliter l'analyse. Par la suite, nous avons regroupé les informations par thèmes car cela permet de structurer les récits et de dégager la fréquence des thèmes manifestes repérables dans les récits.

Enfin, nous avons fait une analyse des contenus manifestes, ultimes révélateurs du sens exacte du phénomène étudié, et des contenus latents car selon L'Ecuyer (1990), elle permet d'accéder au sens caché potentiellement véhiculé par les informations.

\section{Résultats}

2.1. Les représentations sociales relatives à la culture de l'arbre et à l'exploitation forestière fondent l'agroforêt à base de cacaoyers

\subsubsection{Un précédent cacaocultural hérité}

Les paysans rencontrés nous ont dit qu'ils ont hérité des exploitations cacaoyères (Kpangui, 2015). De même, il est vrai que ceux-ci créent de nouvelles exploitations de cacaoyers mais, ils utilisent et adoptent les techniques ancestrales qui fondent l'agroforesterie. D'ailleurs, dans les environs de Kokumbo, la pratique de l'agroforesterie est une pratique ancienne selon les personnes interviewées, cette pratique remonte aux années 1958.

Le précédent cacaocultural hérité est très prononcé dans les pratiques cacaoculturales mises en œuvre. L'agroforesterie semble être ancrée dans le quotidien de ces paysans. Ainsi, les pratiques enseignées par l'Agence Nationale pour le Développement Rural (ANADER) et la structure internationale d'encadrement des paysans BIOPARTENAIRE ne sont-ils pas contraires aux savoirs traditionnels de l'association des arbres d'avec le cacaoyer que leurs ancêtres leur ont légué.

Ces enseignements à partir des outils comme Champ-école renforcent les savoirs locaux. D'ailleurs, un cacaoculteur à Niamkey-Konankro nous a dit ce qui suit: "Nos parents gardaient toujours des arbres dans le cacao...nous aussi, on laisse, on coupe ce qui nous fatigue beaucoup, mais on garde toujours arbre dans cacao, sinon ce n'est pas bon mon papa m'a dit cela. Dieu merci, champ-école nous enseigne même chose, pour avoir un champ certifié on doit laisser au minimum 18 arbres sur un hectare de champ ». 


\subsubsection{Une perception sacrée de l'Allah et de quelques arbres associés}

Les paysans accordent une importance à l'arbre autant dans la forêt que dans leurs exploitations cacaoyères. L'arbre est très important dans la société baoulé, surtout le «Allah» connu sous le nom commun d'iroko. Les perceptions qui relèvent de ce grand arbre soulignent l'importance qu'il revêt dans la culture dans la périphérie de Kokumbo. L'iroko est un arbre qu'il ne faut surtout pas abattre dans un champ de cacaoyers. La divinisation de l'arbre Allah est bien vivace dans les communautés locales. Le poids culturel n'est pas relevé depuis nos investigations car elle est ancrée dans la tradition baoulé.

Les prénoms ALLAH dans la nomenclature des noms baoulé montrent bien cet arbre demeure particulier. Selon les cacaoculteurs, l'iroko favorise la stabilité mystique du champ tout comme son atout biologique. En outre, il est important de rappeler que les arbres peuvent acquérir une perception particulière si des cômians ou féticheurs demandent à un paysan de diviniser un tel arbre car représentant le conjurateur d'un mal ou malheur familial. L'arbre simple, sans antécédent cultuel acquiert une sacralisation. Le fromager, le fraké obtiennent un poids cultuel dans la famille. En somme, l'arbre participe à la vie du baoulé de Niamkey-Konankro (Photo 2).

\subsubsection{Les divers usages socio-économiques et culturels des arbres associés}

Le Ceiba pentandra (ou Gnin) est un arbre imposant, les jeunes feuilles sont consommées comme sauce par les personnes locales. Au plan thérapeutique, il est réputé traiter le mal de tête, les vertiges, la constipation, les troubles mentaux, les yeux et la fièvre. Les paysans approuvent également son ombrage modéré dans les champs de cacaoyers (Photo 3).

Elaeis guineensis (ou le M'mé) a des multiples usages ; cette plante est principalement utilisée comme aliment, médicament et boisson alcoolisée. Il occupe une place importante dans la vie sociale des communautés locales. D'après les paysans interrogés, ce sont ses multiples usages qui font qu'il est conservé dans le champ de cacao. 


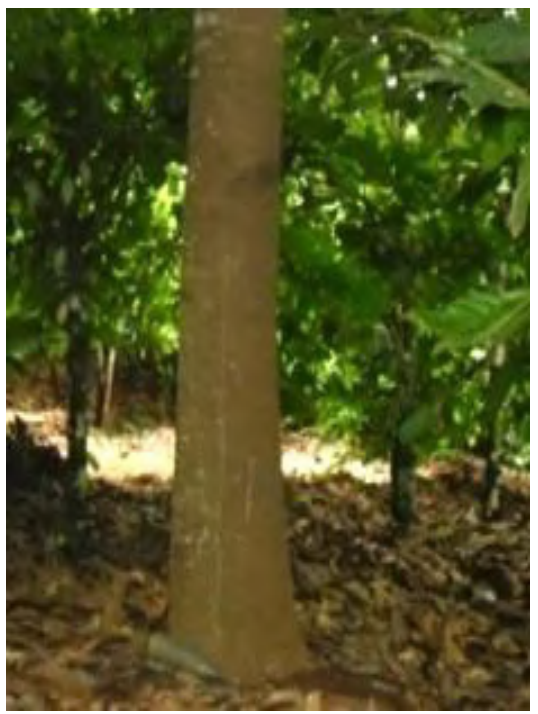

Photo 2. Vue de Milicia excelsa ou Allah en langue baoulé à Niamkey-Konankro, Centre de la Côte d'Ivoire

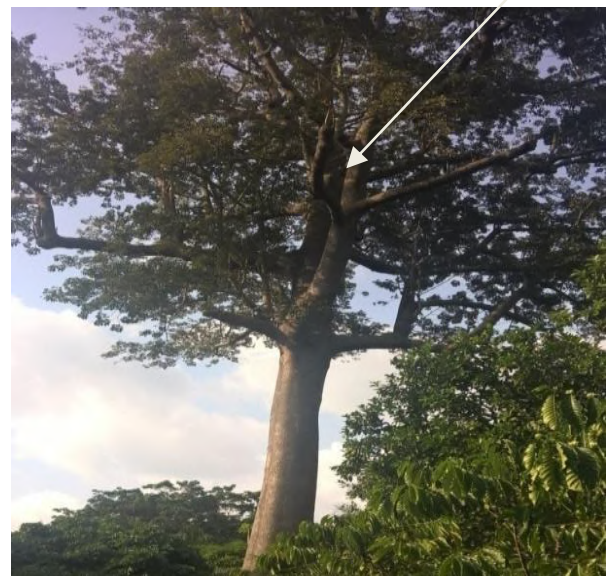

Photo 3. Vue de Ceiba pentandra ou le Gnin en langue baoulé à Niamkey-Konankro, Centre de la Côte d'Ivoire

Par contre, la surabondance de cette plante impacte négativement sur la plantation. Pour les paysans, Elaeis guineensis a plus d'atouts que d'inconvénients comme le répète un des paysans répondant à nos questions : "M'mé fait beaucoup de choses dans les champs, tout est bon dans M'mé là, mé fait huile rouge, M'mé fait n'zan (alcool) avec la sève, M'mé fait balai, panier, M'mé fait champignon » (Photos 4 et 5). 


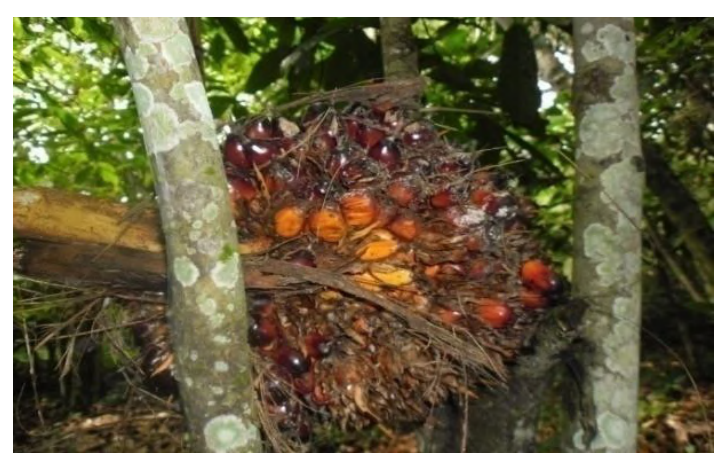

Photo 4. Vue d'un régime de graines d'Elaeis guineensis

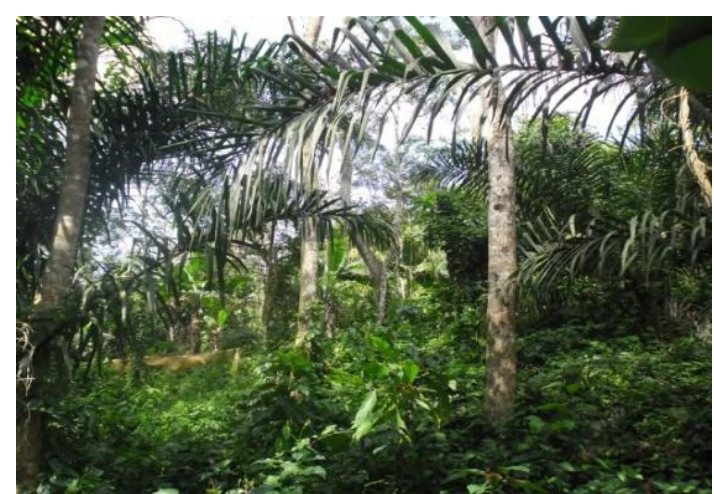

Photo 5. Vue des palmes d'Elaeis guineensis

Le Spathodea campanulata (ou bibissiri) est présent dans toutes les exploitations agroforestières visitées. Les planteurs lui reconnaissent de nombreuses valeurs notamment un ombrage satisfaisant et favorisant l'humidité du cacaoyer. On l'utilise également dans la pharmacopée locale (Photo 6).

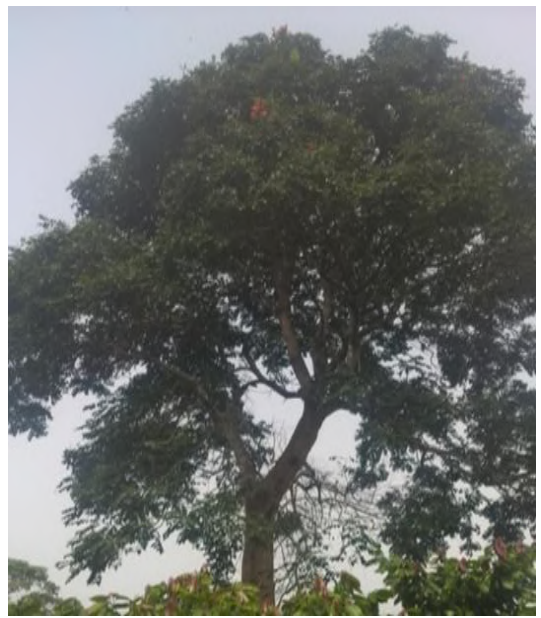

Photo 6. Vue de Spathodea campanulata ou le bibissiri en langue baoulé à NiamkeyKonankro, Centre de Côte d'Ivoire 


\subsection{Les institutions traditionnelles de régulation agricole et un mode d'accès à la terre uniquement autochtone participent au maintien de leur choix cacaocultural}

\subsubsection{Une modalité d'accès à la terre uniquement réservée aux autochtones}

D'après les enquêtes faites, le mode d'accès à la terre à Langossou et Niamkey-Konankro est très restrictif. En effet, aucun individu en dehors de l'autochtone baoulé ne peut acquérir une parcelle de terres cultivables. Pour les locaux, la vente des terres à une tierce relève non seulement de la fainéantise mais aussi expose les générations à venir à des conflits d'héritage entre acheteurs et autochtones héritiers des biens du vendeur de parcelles.

La vente des terres crée beaucoup de problèmes dans les familles. C'est pour cette raison que les populations locales ont choisi de restreindre le mode d'accès au foncier aux seuls autochtones. En outre, cette modalité d'accès à la terre influe sur la migration agricole car la migration est marginale. La pression sur les exploitations cacaoyères s'en trouve repoussée car les migrants agricoles ont cette attitude à adopter des pratiques agricoles pour des rendements très productifs immédiats et à court terme.

\subsection{Une régulation périodique des activités agricoles}

La tradition locale a un plan de régulation des activités champêtres (Tableau 1). Tout individu connaît les jours de champ et les jours non ouvrables pour les activités champêtres. En effet, la tradition n'admet pas que l'on puisse faire ce qu'on veut. Que l'on soit chrétien ou pas, pendant les jours non ouvrables, les pratiques défendues demeurent le travail du paysan qui donne « sueur » c'est-à-dire le sarclage, les buttes, la chasse etc. En outre, les activités tolérées sont la coupe de bois, l'extraction de boissons locales et le transport de vivriers pour la consommation domestique.

Si formellement, personne n'est désignée pour la dénonciation de ces restrictions, il n'en demeure pas moins que la conscience collective soit interpellée pour éviter la famine et/ ou baisse de la productivité. Pour ce faire, tout le monde doit dénoncer une transgression agricole. En effet, pour un gardien de la tradition "il y a toujours quelqu'un qui te voit ». Chez la femme habitant dans les environs de Kokumbo, les restrictions s'étendent pendant ses jours de menstruation. Elle apparaît impure pour aller au champ. Les maladies, les décès, les amendes, les morsures de serpent représentent cette panoplie de sanctions à laquelle s'exposent ceux ou celles qui ne font pas sienne la régulation calendaire. Les paysans acquiescent : c'est comme ça, il faut laisser comme ça! 


\subsection{La promotion agroforestière initiée par l'Etat et les acteurs environnementaux engraisse la continuation de l'agroforesterie à base de cacaoyers.}

\subsubsection{L'engagement de l'Etat}

Depuis octobre 2012, la redynamisation durable du secteur du cacao a été lancée par le Gouvernement à travers la création du Conseil CaféCacao (CCC). Des actions d'informations, de sensibilisation et de formation sont exécutées principalement dans les environs de Kokumbo par deux structures dont l'une est l'ANADER (Agence Nationale d'Aide au Développement Rural engagée par l'Etat ivoirien et l'autre privée est BIOPARTENAIRE. Les outils comme «Champ-école-Paysan» au niveau de l'ANADER et des modules de formations proposées aux paysans par Biopartenaire participent à la production d'un cacao durable (Photo 7).

Tableau I. Périodes de régulation des activités agricoles dans les villages de Niamkey-

Konankro et Langossou, notre enquête juin 2016

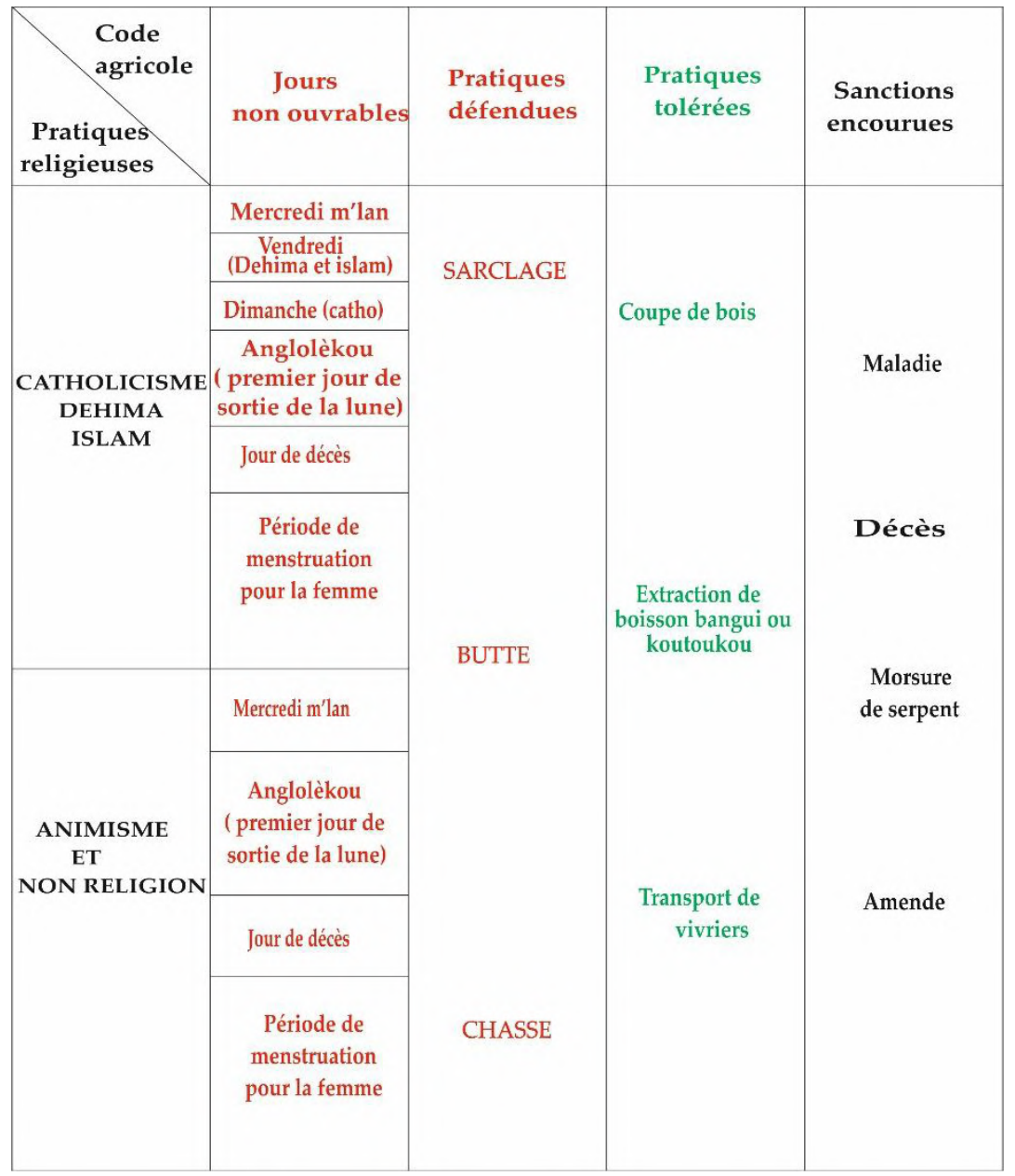




\subsubsection{L'acceptabilité sociale}

Les paysans ont une bonne perception de ces initiatives étatiques car le partenariat est gagnant-gagnant. En effet, si l'Etat veut labelliser sa production cacaoyère en production durable, le seul bénéficie pour les paysans vient du fait de leurs affiliations en coopératives qui leur permettent d'avoir des ristournes, des pesticides et autres avantages.

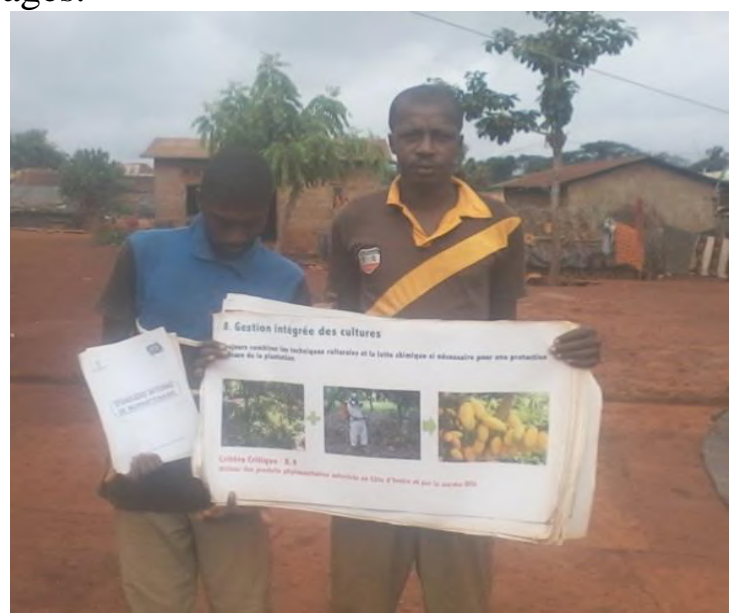

Photo 7. Vue des paysans présentant des modules de formation de la structure Biopartenaire à Langossou, Centre de Côte d'Ivoire

\section{Discussion}

Nous avons vu que les fondements socioculturels des arbres associés dans les exploitations cacaoyères dans les villages de Niamkey-Konankro et de Langossou sont essentiels dans le choix de la pratique agroforestière. D'autres auteurs ont étayé cela dans notre site d'étude. En effet, le paysan habitant les villages de Niamkey-Konankro et de Langossou conserve les espèces par ce que pour lui, l'arbre participe à l'équilibre socioculturel et agronomique de sa plantation.

Cette situation est similaire aux observations de plusieurs auteurs dont Adou Yao et al. (2015) qui constatent que les espèces associés aux cacaoyers rentrent dans l'alimentation, le bois de chauffe et dans la médecine. Ces espèces sont généralement, des espèces locales issues de la régénération naturelle et laissées délibérément pour leurs usages et le rôle qu'elles jouent dans l'exploitation. Aussi, pour plusieurs raisons (alimentaire, médicinale, culturelle), certains exploitants introduisent volontairement en plus des espèces locales, des espèces exotiques originaires d'Asie et d'Amérique dans leur exploitation, pratique rapportée également par Kpangui et al.(2015) en Côte d'Ivoire, par Asare et al.(2009) au Ghana, par Oke et Odebiyi (2007) au Nigeria, par Sonwa et al.(2007) et par Zapfack et al. (2002) au Cameroun. Dans ces mêmes localités de Langossou et de Niamkey-Konankro 
(département de Toumodi, Centre Côte d'Ivoire), Kouassi (2014) a évalué l'importance financière des espèces alimentaires présentes dans les exploitations de cacaoyers. Pour lui, la diversification des cultures apporte une plus-value des revenus.

Il revient que la pratique agroforestière à Niamkey-Konankro tout comme à Langossou est dictée par la conformité aux habitudes et coutumes agricoles. C'est pourquoi, les informations recueillies dans le cadre de cette étude nous permettent de dire que la pratique agroforestière à base de cacao résulte des «habitus cacaoculturaux». En effet, «les sociétés traditionnelles africaines en général sont régies par des lois sociales qui se transmettent de génération en génération » (Koné, 2012). En outre, il est à relever que l'accès à la terre à Niamkey-Konankro et à Langossou exclusivement réservé aux autochtones met en exergue l'émergence d'une communauté autochtone homogène. En effet, Freud et al. (2000) ont souligné que la migration est un facteur d'abattage des arbres. C'est la raison pour laquelle la pression relative à l'abattage immédiat des arbres dans les exploitations cacaoyères à l'effet de rendements économiques à court terme se retrouve être repoussée des exploitations cacaoyères.

Dans un contexte favorable au maintien des agroforêts à base de cacao, un autre facteur vient en aval «stabiliser » la pratique déjà connue et pratiquée. Il s'agit de la promotion agroforestière des plantations promue par le Gouvernement ivoirien. En effet, la mise en place du conseil café cacao depuis octobre 2012 exige désormais la production de façon durable d'un cacao de qualité.

\section{Conclusion}

$\mathrm{Au}$ terme de cette étude, fort est de constater que la pratique agroforestière à base de cacaoyers dans les villages de Niamkey-Konankro et de Langossou a de beaux jours devant elle. En effet, le contexte culturel et politique dynamise sa continuation. Dans une optique idéal-typique, nous avons pu déterminer un profil agroforestier local de notre pays. En somme, la pratique de l'agroforesterie dans la zone de Kokumbo est modifiée par des forces externes et internes qui la sous-tendent et, donc protègent sa durabilité (Figure 2). 


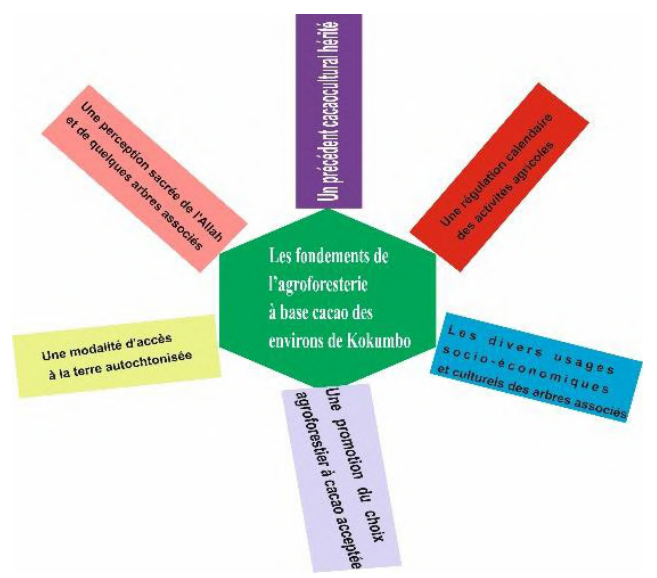

Figure 2. Schématisation des fondements de l'agroforesterie à base de cacaoyers dans les environs de Kokumbo : un profil agroforestier ivoirien, notre enquête juin 2016.

\section{References:}

1. Adou Yao, C.Y., Kpangui, K.B., Koffi, B. J. C., \& Vroh, B. T. A. (2015). Farming practices, diversity and utilizations of associated species of cocoa plantations in a forest savannah transition zone, Center Côte d'Ivoire. Global Journal of Wood Science, Forestry and Wildlife. 3 (3): 094-100.

2. Adou Yao, C.Y., Kpangui, K. B., Vroh, B. T. A. \& Ouattara, D. (2016). Pratiques culturales, valeurs d'usage et perception des paysans des espèces compagnes du cacaoyer dans des agroforêts traditionnelles au centre de la Côte d'Ivoire, Revue d'ethnoécologie, DOI : 10.4000/ethnoécologie.2474, 9, 17p.

3. Aktouf, O. (1987). Méthodologie des sciences et approche qualitative des Organisations : une introduction à la démarche classique et une classique et critique, Canada, Presse Universitaire de Québec, 213p.

4. Bourdieu, P. (1980). Le sens pratique, Paris, édition Minuit, 480 p.

5. Freud, E.H., Petithuguenin, P., \& Richard, J. (2000). Les champs du cacao : un défi pour la compétitivité Afrique Asie, Paris, Karthala et Cirad, Paris 2000, $207 \mathrm{p}$.

6. ICCO (2008). Rapport annuel 2008/2009. London WC1A 1NU, United Kingdom, 42 p.

7. Koffi, N.J. (2006). Kokumbo, un village du canton fahafouè dans le département de Toumodi : des origines à nos jours ; Mini-mémoire de Licence, Histoire moderne et contemporaine, Université Félix Houphouët-Boigny de Cocody-Abidjan, Côte d'Ivoire, 33 p.

8. Koné, W.M., Azokou, A., \& Bakayoko, A. (2012). Preliminary Study on Polysaccharides and certain secondary metabolites of medicinal 
plants used in Côte d'Ivoire for wound healing, Research Journal of Medicinal Plants. 6 (3): 214-224.

9. Kouassi, D.F. (2013). Caractérisation des agroforêts à cacao : évaluation des performances agronomiques et économiques ; Mémoire de Master I, UFR Biosciences, Université Félix Houphouët-Boigny de Cocody-Abidjan, Côte d'Ivoire, $50 \mathrm{p}$.

10. Kpangui, K.B. (2015). Dynamique, diversité végétale et valeurs écologiques des agroforêts à base de cacaoyers dans le Centre de la Côte d'Ivoire: cas de la Sous-préfecture de Kokumbo, Thèse de Doctorat unique, UFR Biosciences, Université Félix HouphouëtBoigny de Cocody-Abidjan, Côte d'Ivoire, $187 \mathrm{p}$.

11. L'Écuyer, R. (1990). Méthodologie de l'analyse développementale de contenu. Méthode GPS et concept de soi, Québec, Presse Universitaire de Québec, 472 p.

12. Sonwa, D., Weise, S.F., Tchatat, M., Nkongmeneck, B., Adesina, A.A., Ndoye, O., \& Gockowski, J. (2001). Rôle des agroforêts à cacao dans la foresterie paysanne et communautaire au sud-Cameroun. Document RDFN, n²5 g (i), 12 p. 